\title{
Participação do Brasil nos Jogos Paraolímpicos de Sydney: apresentação e análise
}

\author{
Alberto Martins da Costa ${ }^{1}$ e Sílvio Soares dos Santos ${ }^{2}$
}

\section{RESUMO}

Na Paraolimpíada de Sydney, o Brasil demonstrou que pode ser mais do que um mero participante, apresentandose de forma brilhante diante do mundo do desporto para pessoas portadoras de deficiências. No entanto, mostrou, também, que muitas coisas ainda podem e devem ser acertadas no seu aspecto técnico e administrativo. O Brasil apresentou uma grande evolução do seu paradesporto, assim como a sua grande capacidade de se tornar uma potência no desporto adaptado mundial. Com o objetivo de expor concreta e cientificamente esta capacidade é que mostramos e comentamos, através de dados estatísticos, um panorama dos países participantes dos Jogos Paraolímpicos de Sydney 2000, o quadro geral de medalhas e a participação brasileira nas várias modalidades. Analisamos, ainda, no sentido de verificar a evolução da performance qualitativa dos atletas brasileiros, uma comparação com a sua última participação nos Jogos Paraolímpicos de Atlanta 1996.

Palavras-chave: Performance. Medalhas. Atletas paraolímpicos.

\section{ABSTRACT}

\section{Brazilian participation in the Paralympic Games of Syd- ney: presentation and analysis}

In the Sydney Paralympic Games, the Brazilian Team demonstrated that can be more than just another competitor. The Brazilian Paralympic Team exhibited excellence

1. Professor Adjunto IV, Faculdade de Educação Física (FAEFI), Universidade Federal de Uberlândia (UFU).

2. Professor Adjunto I, Faculdade de Educação Física (FAEFI), Universidade Federal de Uberlândia (UFU).

Submetido em: 4/4/02

Versão revisada recebida em: 1/5/02

Aceito em: 19/5/02

Endereço para correspondência:

Núcleo Interdisciplinar de Estudos e Pesquisas em Atividade Física e

Saúde da Universidade Federal de Uberlândia (NIAFS/UFU)

Rua Beijamin Cosntant, 1.286 - Aparecida

38406-039 - Uberlândia, MG

E-mail: amcosta@ufu.br to the world of sports for the disabled. However, there were a lot of things that need technical and administrative improvement. Brazil showed a great evolution in parasports, and a great capacity to become one of the world powers of adapted sports. The aim of this work is to make a real and scientific presentation of such capacity, by means of statistics of the countries participating in the 2000 Sydney Paralympic Games, with the general results of medals, and the Brazilian participation in different modalities. An analysis is made of the qualitative performance evolution of Brazilian athletes through a comparison to their prior participation in the 1996 Atlanta Paralympic Games.

Key words: Performance. Medals. Sydney Paralympic Games.

\section{INTRODUÇÃO}

O desporto das pessoas portadoras de deficiência, no Brasil de hoje, é uma realidade incontestável, contudo, além das dificuldades encontradas no que diz respeito a infraestrutura dos clubes e associações de esportes, para o desenvolvimento da prática esportiva, tais como: locais apropriados, materiais e equipamentos adequados, além da conscientização dos próprios portadores de deficiência e sua família sobre a importância do esporte como fator preponderante no auxílio à reinserção do portador de deficiência na vida social, a falta de profissionais habilitados e adequadamente preparados para atuarem com essas pessoas nos esportes específicos, principalmente, no que diz respeito a avaliação e metodologia do treinamento esportivo com bases científicas, têm sido, uma das principais queixas e reivindicações dos clubes, associações nacionais, atletas e dirigentes do desporto adaptado no nosso país.

Desde 1996, o Comitê Paraolímpico Brasileiro, despertou para a necessidade de envolver os profissionais das universidades no programa de avaliação e treinamento dos atletas paraolímpicos com vistas à sua participação nas olimpíadas de Atlanta. Nesta ocasião, um programa completo de avaliação médica, laboratorial e da condição físi$\mathrm{ca}$, foi desenvolvido no intuito de subsidiar o treinamento e a preparação final dos atletas convocados para a paraolimpíada de Atlanta. Tendo em vista os resultados obtidos 
em Atlanta, considerados satisfatórios, novamente, em 2000, o Comitê Paraolímpico Brasileiro, convida uma equipe de avaliação para realizar o trabalho com a seleção paraolímpica do Brasil com vistas à sua preparação para participar nos jogos de Sydney 2000, cujas avaliações foram relatadas anteriormente por cada equipe de avaliadores.

O desporto adaptado, no Brasil, chegou num momento de grande decisão política com relação ao seu destino, ou toma-se a decisão de se adequar à realidade internacional no que diz respeito à profissionalização de ações e efetiva participação dos métodos científicos de avaliação e treinamento dos nossos atletas e equipes paraolímpicas para que possamos ingressar no rol das potências esportivas, ou continuamos o nosso papel de sensibilização e adaptação da realidade, nos contentando a sermos meros participantes nos eventos esportivos internacionais.

O Brasil, na Paraolimpíada de Sydney, mostrou que pode ser mais do que um mero participante, ele demonstrou o seu potencial e a sua grande capacidade em se tornar uma potência no desporto adaptado. Com o objetivo de expor concreta e cientificamente esta capacidade é que passamos a mostrar e comentar, a seguir, através de dados estatísticos, a participação brasileira nas várias modalidades em que participou nos Jogos Paraolímpicos de Sydney 2000, assim como, uma comparação com a sua última participação nos Jogos Paraolímpicos de Atlanta 1996.

Nos Jogos Paraolímpicos de Sydney 2000, participaram 128 países com um total aproximado de 4.000 atletas disputando 20 modalidades esportivas, se tornando, assim, o maior evento esportivo de portadores de deficiência até então realizado.

\section{A PARTICIPAÇÃO BRASILEIRA}

O Brasil participou nos Jogos Paraolímpicos de Sydney, em sete modalidades esportivas individuais a saber: natação, atletismo, ciclismo, esgrima, tênis de mesa, halterofilismo e judô e em duas modalidades coletivas: basquete para deficientes mentais e futebol para paralisados cerebrais. Na natação competiu nas provas de $50 \mathrm{~m}, 100 \mathrm{~m}$ e $400 \mathrm{~m}$ nado livre (L), 50 e $100 \mathrm{~m}$ nado costas (C), $50 \mathrm{~m}$ e $100 \mathrm{~m}$ nado peito $(\mathrm{P}), 150 \mathrm{~m}$ e $200 \mathrm{~m}$ metros medley $(\mathrm{M})$, $50 \mathrm{~m}$ e $100 \mathrm{~m}$ nado borboleta (B) além de quatro revezamentos estilos medley e livre. Na modalidade de atletismo participamos nas corridas de $100 \mathrm{~m}, 200 \mathrm{~m}, 400 \mathrm{~m}, 5.000 \mathrm{~m}$, $10.000 \mathrm{~m}$ e na maratona. Nas provas de arremessos participamos nos arremessos de peso, dardo e disco. No ciclismo participamos das provas de $1 \mathrm{~km}, 4 \mathrm{~km}, 63,7 \mathrm{~km}$ e $72,8 \mathrm{~km}$.

Foram conseguidas 22 medalhas sendo seis de ouro, 10 de prata e seis de bronze. Foi uma excelente performance se considerado o número de modalidades individuais sete e o número total de medalhas obtidas nessas modalidades
21 em média três medalhas por modalidade. Das duas modalidades coletivas, uma foi medalhista de bronze. A seguir, apresentaremos a tabela 1 , onde buscamos comparar a participação do Brasil nos Jogos Paraolímpicos de Atlanta com a participação em Sydney.

A participação brasileira em Sydney pode ser considerada como a melhor campanha do Brasil em Jogos Paraolímpicos, pois, se compararmos com a campanha de Atlanta em 1996, no quadro Geral de medalhas, o Brasil se colocou em 37o lugar com 21 medalhas. Em Sydney, o Brasil se posicionou em 24ㅇ lugar no quadro geral de medalhas com 22 medalhas. Contudo, se observarmos a tabela 2 , podemos verificar que houve uma evolução no que diz respeito à qualidade, pois, em Atlanta, foram duas de ouro, seis de prata e 13 de bronze e em Sydney, seis de ouro, 10 de prata e seis de bronze.

Ao contrário do que ocorreu em Atlanta, quando o Atletismo conseguiu o maior número de medalhas: cinco de prata e seis de bronze, a natação foi a modalidade paraolímpica que mais medalhas ganhou nos Jogos de Sydney. No total foram 12 medalhas, sendo uma de ouro, sete de prata e quatro de bronze. Do total de medalhas conseguidas duas de prata e uma de bronze foram conseguidas em provas de revezamento.

Ao analisarmos estes números de forma mais objetiva, podemos observar que os atletas brasileiros, por oito vezes obtiveram a chance concreta de disputar a medalha de ouro, logrando êxito em apenas uma delas. Contudo, quando analisarmos os dados demonstrados nas tabelas abaixo, podemos concluir que em muitos destes casos a diferença entre os atletas do Brasil e o vencedor da prova é quase que insignificante, nos demonstrando que apesar de todas as dificuldades apresentadas durante a fase de preparação da equipe, a mesma não se distanciou substancialmente dos melhores atletas paraolímpicos do mundo.

Assim, passaremos a demonstrar através das tabelas abaixo os resultados obtidos pelos atletas brasileiros nas diferentes modalidades e provas disputadas, comparando o seu resultado com o resultado do campeão da prova.

\section{NATAÇÃO FEMININA}

A tabela 2 mostra os resultados das atletas da natação feminina com o tempo obtido nas provas, sua classificação

TABELA 1

Quadro de medalhas comparativo da participação brasileira nos Jogos Paraolímpicos de Atlanta 1996 e Sydney 2000

\begin{tabular}{lcccc}
\hline Brasil & Ouro & Prata & Bronze & Total \\
Atlanta & 2 & 6 & 13 & 21 \\
Sydney & 6 & 10 & 6 & 22 \\
\hline
\end{tabular}


TABELA 2

Provas de natação feminina

\begin{tabular}{lllcrrr} 
Nome & Prova & Classe & $\begin{array}{c}\text { Tempo } \\
\text { (seg) }\end{array}$ & Classif. & $\begin{array}{c}\text { Vencedor } \\
\text { (seg) }\end{array}$ & \multicolumn{1}{c}{$\begin{array}{c}\text { Dif. } \\
\text { (\%) }\end{array}$} \\
\hline C.S. & $50 \mathrm{C}$ & S4 & 72,9 & 10 & 50,92 & 30,2 \\
C.S. & $100 \mathrm{~L}$ & S4 & 149,81 & 12 & 96,27 & 35,7 \\
C.S. & $50 \mathrm{~L}$ & $\mathrm{~S} 4$ & 70,78 & 13 & 40,64 & 42,6 \\
\hline F.S. & $50 \mathrm{~L}$ & $\mathrm{~S} 11$ & 33,51 & $\mathbf{1}$ & 33,51 & 0,0 \\
F.S. & $100 \mathrm{~L}$ & $\mathrm{~S} 11$ & 77,55 & 5 & 74,31 & 4,2 \\
\hline R.F. & $50 \mathrm{P}$ & $\mathrm{SB} 3$ & 75,08 & 7 & 58,12 & 22,6 \\
R.F. & $150 \mathrm{M}$ & $\mathrm{SM} 4$ & 244,7 & 12 & 177,31 & 27,5 \\
\hline
\end{tabular}

final, o tempo da vencedora da prova e a diferença percentual entre os tempos obtidos por ambas.

Como podemos observar na tabela acima, a distância que separa as atletas R.F. e C.S. em relação às atletas medalhistas de ouro foi em média $31,7 \%$. Essa é uma cifra relativamente alta, o que nos comprova, que a performance destas atletas está aquém do desejado.

Com relação à atleta F.S. a diferença apresentada é de $4,2 \%$ em relação ao melhor tempo da prova dos $100 \mathrm{~m}$ e zero na prova dos $50 \mathrm{~m}$ livre, já que ela foi a vencedora da prova.

$\mathrm{Na}$ prova dos $50 \mathrm{~m}$ nado livre ela apresentou o menor tempo de saída $(8,77 \mathrm{~s})$ dentre todas as concorrentes além de uma freqüência média de 56,6 ciclos/min, um comprimento médio de braçadas de $1,51 \mathrm{~m}$ e uma velocidade média de $1,42 \mathrm{~m} / \mathrm{s}$. Ela percorreu os 25 metros iniciais em $15,47 \mathrm{~s}$ e os 25 metros finais em 18,04s. Houve uma queda significativa de aproximadamente $14 \%$ entre a primeira e a segunda parte da prova. Na prova dos 100 metros nado livre ela manteve a excelência na saída $(9,07 \mathrm{~s})$ saindo mais rápido que todas as outras concorrentes (aproximadamente $8,9 \%$ melhor que a primeira colocada), no entanto, cerca de $3,3 \%$ pior que na prova dos $50 \mathrm{~m}$ livre. Nessa prova o primeiro quarto foi percorrido em $16,58 \mathrm{~s}$, o segundo quarto em 19,34 s, o terceiro quarto em 20,24 s e a última parte em 21,39s. Os comprimentos de braçada foram de $1,45 \mathrm{~m}$, $1,63 \mathrm{~m}, 1,48 \mathrm{~m}$ e $1,56 \mathrm{~m}$ respectivamente do primeiro ao último quarto da prova. A freqüência dos ciclos de braçadas por minuto foram de $55,3 \mathrm{~s}, 48,7 \mathrm{~s}, 47,5 \mathrm{~s}$ e 44,3 . Quando comparada com a ganhadora da prova pode-se verificar que o comprimento de braçada da atleta Fabiana encontra-se dentro de patamares ótimos, durante toda a prova. No entanto a freqüência de braçadas foi em torno de $11,1 \%$ menor durante toda a prova que a atleta vencedora da prova. Em relação à virada o tempo gasto por Fabiana foi aproximadamente $5,6 \%$ pior que a atleta vencedora da prova.
Em resumo podemos concluir que a vantagem de 5,8\% no parâmetro comprimento de braçada em favor da atleta F.S. assim como a boa saída (ganho de $8,9 \%$ ) não foram suficientes para contrabalançar as perdas ocorridas na freqüência de braçada $(11,1 \%)$ e no tempo de virada $(5,6 \%)$. As quedas sistemáticas do tempo de percurso durante a prova dos 100 metros nado livre podem ser portanto, atribuídas em sua grande parte, à queda na frequiência de braçadas durante a prova. Por outro lado se observarmos a média de sua participação, podemos afirmar que a mesma se posicionou muito bem nas provas que disputou ( $1^{\underline{a}}$ e $5^{\underline{a}}$ colocações).

A equipe de natação feminina foi muito reduzida nesses jogos. Ela foi composta por três atletas e ganhou uma medalha fazendo em média 0,33 medalha por atleta. Quanto ao número de provas disputadas sete foi obtida uma medalha a cada sete provas ou em média 0,14 medalha por prova disputada.

\section{NATAÇÃO MASCULINA}

Na tabela 3, apresentamos os resultados dos atletas da equipe masculina de natação com o tempo obtido nas provas, sua classificação final, o tempo do vencedor da prova e a diferença percentual entre os tempos obtidos por ambos.

A primeira observação que podemos apontar com relação a participação geral, diz respeito ao número de atletas e o número de medalhas conseguidas. A equipe masculina com 12 nadadores ganhou 11 medalhas perfazendo em média 0,92 medalha por nadador. Em relação ao número de provas disputadas (43) foi obtida uma medalha a cada 3,9 provas disputadas ou 0,26 medalha por prova. No que respeito a avaliação geral da equipe, os dados apresentados nos permite afirmar que a equipe masculina apresentou, de uma forma geral, um bom desempenho nas provas em que disputou, principalmente se considerarmos que por sete vezes obteve a chance real de conseguir a medalha de ouro. No entanto, acreditamos ser oportuno a realização futura de uma avaliação do comportamento individual de cada atleta nas suas respectivas provas para que possamos analisar a sua performance no decorrer da prova, possibilitando uma inferência mais segura quanto a escolha da prova e do treinamento adequado para a sua realização.

\section{ATLETISMO}

Ao analisarmos a tabela 4, podemos constatar que há uma necessidade de melhor orientação dos atletas brasileiros nas respectivas provas, pois com a evolução da performance esportiva paraolímpica internacional, faz-se necessário que os atletas tenham claramente definido sua prova 


\begin{tabular}{|c|c|c|c|c|c|c|}
\hline \multicolumn{7}{|c|}{$\begin{array}{l}\text { TABELA } 3 \\
\text { Equipe de natação masculina paraolímpica }\end{array}$} \\
\hline Nome & Prova & Classe & $\begin{array}{l}\text { Tempo } \\
\text { (seg) }\end{array}$ & Classif. & $\begin{array}{l}\text { Vencedor } \\
\text { (seg) }\end{array}$ & $\begin{array}{l}\text { Dif. } \\
(\%)\end{array}$ \\
\hline A.G. & $100 \mathrm{~L}$ & S6 & 70,6 & 2 & 68,1 & 3,5 \\
\hline A.G. & $50 \mathrm{~L}$ & S6 & 32,25 & 2 & 32,13 & 0,4 \\
\hline A.G. & $50 \mathrm{~L}$ & S6 & 32,41 & 4 & 31,31 & 3,4 \\
\hline A.G. & $200 \mathrm{M}$ & SM6 & 191,73 & 5 & 177,42 & 7,5 \\
\hline A.P. & $100 \mathrm{~L}$ & S2 & 170,59 & 9 & 60,67 & 64,4 \\
\hline A.P. & $50 \mathrm{C}$ & S2 & 84,84 & 10 & 65,88 & 22,3 \\
\hline A.P. & $50 \mathrm{~L}$ & $\mathrm{~S} 2$ & 82,59 & 11 & 60,36 & 26,9 \\
\hline C.S. & $100 \mathrm{~L}$ & S4 & 92,17 & 2 & 85,92 & 6,8 \\
\hline C.S. & $50 \mathrm{~L}$ & S4 & 41,62 & 3 & 38,84 & 6,7 \\
\hline C.S. & $200 \mathrm{~L}$ & S4 & 208,47 & 4 & 182,19 & 12,6 \\
\hline D.G. & $50 \mathrm{~L}$ & S10 & 25,89 & 3 & 25,37 & 2,0 \\
\hline D.G. & $100 \mathrm{~L}$ & S10 & 58,45 & 7 & 54,3 & 7,1 \\
\hline F.M. & $400 \mathrm{~L}$ & S9 & 289,3 & 7 & 265,73 & 8,1 \\
\hline F.M. & $100 \mathrm{~B}$ & S9 & 74,31 & 13 & 64,58 & 13,1 \\
\hline F.M. & $100 \mathrm{~L}$ & S9 & 63,23 & 13 & 58,62 & 7,3 \\
\hline F.A. & $50 \mathrm{C}$ & S5 & 41,57 & 4 & 39,2 & 5,7 \\
\hline F.A. & $50 \mathrm{~L}$ & S5 & 40,01 & 8 & 34,47 & 13,8 \\
\hline F.A. & $100 \mathrm{P}$ & SB4 & 115,48 & 8 & 97,37 & 15,7 \\
\hline F.A. & $100 \mathrm{~L}$ & S5 & 95,25 & 11 & 77,94 & 18,2 \\
\hline G.A. & $150 \mathrm{M}$ & SM3 & 214,97 & 3 & 186,44 & 13,3 \\
\hline G.A. & $100 \mathrm{~L}$ & S3 & 126,65 & 6 & 53,42 & 57,8 \\
\hline G.A. & $50 P$ & SB2 & 70,93 & 6 & 60,56 & 14,6 \\
\hline G.S. & $100 \mathrm{C}$ & S8 & 76,73 & 6 & 69,9 & 8,9 \\
\hline G.S. & $100 \mathrm{P}$ & SB6 & 99,81 & 6 & 93,72 & 6,1 \\
\hline G.S. & $200 \mathrm{M}$ & SM7 & 187,45 & 9 & 169,17 & 9,8 \\
\hline G.S. & $100 \mathrm{~L}$ & S8 & 69,25 & 13 & 62,5 & 9,7 \\
\hline G.S. & $50 \mathrm{~L}$ & S8 & 31,37 & 15 & 28,3 & 9,8 \\
\hline I.V. & $200 \mathrm{M}$ & SM5 & 213,08 & 5 & 188,39 & 11,6 \\
\hline I.V. & $100 \mathrm{C}$ & S6 & 94,28 & 10 & 78,14 & 17,1 \\
\hline J.S. & $50 \mathrm{~B}$ & S4 & 62,81 & 6 & 49,98 & 20,4 \\
\hline J.S. & $50 \mathrm{C}$ & S4 & 55,35 & 7 & 48,3 & 12,7 \\
\hline J.S. & $50 \mathrm{~L}$ & S4 & 54,81 & 14 & 38,84 & 29,1 \\
\hline J.S. & $100 \mathrm{~L}$ & S4 & 120,81 & 16 & 85,92 & 28,9 \\
\hline L.S. & $50 \mathrm{~B}$ & S6 & 34,15 & 2 & 34,01 & 0,4 \\
\hline L.S. & $50 \mathrm{~L}$ & S6 & 33,27 & 5 & 32,13 & 3,4 \\
\hline L.S. & $100 \mathrm{~L}$ & S6 & 73,76 & 7 & 68,1 & 7,7 \\
\hline M.B. & $50 \mathrm{~L}$ & S9 & 27,17 & 2 & 26,36 & 3,0 \\
\hline M.B. & $100 \mathrm{~L}$ & S9 & 61,15 & 6 & 58,62 & 4,1 \\
\hline M.B. & $100 \mathrm{C}$ & S9 & 72,49 & 9 & 65,15 & 10,1 \\
\hline Revez. masc. & $4 \times 50 \mathrm{~L}$ & $20 p t$ & 166,77 & 2 & 144,42 & 13,4 \\
\hline Revez. masc. & $4 \times 50 \mathrm{M}$ & $20 p t$ & 164,93 & 2 & 159,94 & 3,0 \\
\hline Revez. masc. & $4 \times 100 \mathrm{~L}$ & $34 \mathrm{pt}$ & 254,69 & 3 & 246,85 & 3,1 \\
\hline Revez. masc. & $4 \times 100 \mathrm{M}$ & $34 p t$ & 312,28 & 7 & 272,39 & 12,8 \\
\hline
\end{tabular}


principal e que para ela se prepare adequadamente. Se observarmos os resultados brasileiros comparando-os com o campeão da respectiva prova, podemos observar nitidamente a compatibilidade do atleta com relação a sua melhor prova, pois, a distância que o separa do primeiro lugar em alguns casos não são tão significativas, bastando para isto, uma melhor avaliação de suas aptidões e uma recomendação científica de treinamento específico de acordo com suas características.

\begin{tabular}{|c|c|c|c|c|c|c|}
\hline \multicolumn{7}{|c|}{$\begin{array}{c}\text { TABELA } 4 \\
\text { Resultados das provas de pista } \\
\text { da equipe masculina de atletismo }\end{array}$} \\
\hline Nome & Prova & Classe & $\begin{array}{c}\text { Tempo } \\
\text { (seg) }\end{array}$ & Classif. & $\begin{array}{c}\text { Vencedor } \\
\text { (seg) }\end{array}$ & $\begin{array}{l}\text { Dif. } \\
(\%)\end{array}$ \\
\hline A.L. & $100 \mathrm{~m}$ & T13 & 11,39 & 2 & 11,36 & 0,3 \\
\hline A.L. & $200 \mathrm{~m}$ & $\mathrm{~T} 13$ & 22,75 & 2 & 22,42 & 1,5 \\
\hline A.L. & $400 m$ & T13 & 58,46 & 9 & 50,64 & 13,4 \\
\hline A.S. & $400 \mathrm{~m}$ & $\mathrm{~T} 46$ & 50,27 & 2 & 50,16 & 0,2 \\
\hline A.S. & $200 \mathrm{~m}$ & T46 & 23,14 & 8 & 22,02 & 4,8 \\
\hline A.S. & $100 \mathrm{~m}$ & $\mathrm{~T} 46$ & 11,6 & 13 & 11,12 & 4,1 \\
\hline Au.S. & Maratona & T12 & 9.705 & 5 & 9191 & 5,3 \\
\hline Au.S. & $10.000 \mathrm{~m}$ & T12 & $2.045,55$ & 6 & $1.922,77$ & 6,0 \\
\hline Au.S. & $5.000 \mathrm{~m}$ & T12 & 970,16 & 7 & 896,47 & 7,6 \\
\hline S.A. & $400 m$ & $\mathrm{~T} 20$ & 52,45 & 8 & 48,7 & 7,1 \\
\hline S.A. & $100 m$ & T20 & 11,73 & 15 & 10,93 & 6,8 \\
\hline
\end{tabular}

TABELA 5

Resultados das provas de pista da equipe feminina de atletismo

\begin{tabular}{lcccccc} 
Nome & Prova & Classe & $\begin{array}{c}\text { Tempo } \\
\text { (seg) }\end{array}$ & Classif. & $\begin{array}{c}\text { Vencedor } \\
\text { (m) }\end{array}$ & $\begin{array}{c}\text { Dif. } \\
\text { (\%) }\end{array}$ \\
\hline Ad.S. & $100 \mathrm{~m}$ & $\mathrm{~T} 12$ & 12,46 & $\mathbf{1}$ & 12,46 & 0,0 \\
Ad.S. & $200 \mathrm{~m}$ & $\mathrm{~T} 11$ & 24,99 & $\mathbf{1}$ & 24,99 & 0,0 \\
Ad.S. & $400 \mathrm{~m}$ & $\mathrm{~T} 11$ & 59,46 & $\mathbf{2}$ & 56,83 & 4,4 \\
\hline M.J. & $200 \mathrm{~m}$ & $\mathrm{~T} 12$ & 26,17 & 4 & 25,38 & 3,0 \\
M.J. & $100 \mathrm{~m}$ & $\mathrm{~T} 12$ & 13,16 & 7 & 12,46 & 5,3 \\
\hline
\end{tabular}

No que se refere aos resultados da equipe feminina, pouco temos a comentar, uma vez que, a equipe brasileira foi composta de apenas duas atletas deficientes visuais, sendo que uma delas foi vitoriosa em duas provas, estabelecendo também nestas duas provas um novo recorde mundial e consagrando-se vice-campeã em outra prova, mas, ao observarmos seu resultado nesta prova, podemos também detectar que esta prova não está de acordo com suas características e aptidões físicas e que a concentração nos seus treinamentos devem ser direcionados de acordo com suas potencialidades atléticas. No que se refere a atleta M.J. acreditamos que também deva ser avaliado suas melhores características e adequá-las para a prova compatível, pois os resultados parecem indicar uma melhor performance na prova dos 200 metros o que poderia também direcionar os seus treinamentos preparando-a para sua melhor prova.

\begin{tabular}{|c|c|c|c|c|c|c|}
\hline \multicolumn{7}{|c|}{$\begin{array}{c}\text { TABELA } 6 \\
\text { Resultados das provas de campo } \\
\text { da equipe masculina de atletismo }\end{array}$} \\
\hline Nome & Prova & Classe & $\begin{array}{l}\text { Distância } \\
\text { (m) }\end{array}$ & Classif. & $\begin{array}{c}\text { Vencedor } \\
\text { (m) }\end{array}$ & $\begin{array}{l}\text { Dif. } \\
(\%)\end{array}$ \\
\hline A.L. & Disco & F37 & 42,97 & 3 & 44,63 & 3,9 \\
\hline A.L. & Dardo & F37 & 35,05 & 9 & 43,84 & 25,1 \\
\hline
\end{tabular}

No Brasil, as provas de campo do atletismo, são as provas mais disputadas e as que apresentam um maior número de competidores nos campeonatos regionais e nacionais, apesar disto, o nível técnico dos nossos atletas, ainda, não são de nível internacional, pois como podemos observar na tabela acima, a delegação brasileira se fez representar por apenas um atleta masculino nas provas de campo e pelo resultado alcançado, podemos verificar claramente que sua real aptidão está no arremesso de disco, pois, a diferença do seu resultado para o campeão da prova não é significativa, para o que, recomendamos, que seja verificado durante sua futura preparação, um direcionamento tanto físico como técnico específico para a sua verdadeira aptidão, propiciando-lhe condições de melhores chances na prova.

TABELA 7

Resultados das provas de campo da equipe feminina de atletismo

Nome Prova Classe Distância Classif. Vencedor Dif. (m) (m) (\%)

\begin{tabular}{lllrcrr}
\hline E.P. & Peso & F37 & 7,04 & 8 & 8,57 & 21,7 \\
E.P. & Dardo & F37 & 14,66 & 8 & 21,85 & 49,0 \\
\hline R.F. & Disco & F58 & 31,58 & $\mathbf{1}$ & 31,58 & 0,0 \\
R.F. & Peso & F58 & 9 & $\mathbf{1}$ & 9 & 0,0 \\
R.F. & Dardo & F58 & 19,39 & 5 & 25,54 & 31,7 \\
\hline S.G. & Peso & F57 & 6,71 & 4 & 7,85 & 17,0 \\
S.G. & Disco & F58 & 20,16 & 12 & 31,58 & 56,6 \\
S.G. & Dardo & F58 & - & Desq & - & - \\
\hline
\end{tabular}


O Brasil se fez representar nas provas de campo feminina, por três atletas, sendo que uma delas sagrou-se campeã e recordista mundial em duas provas, ficando claro que apesar de biomecanicamente apresentarem movimentos diferentes, a atleta R.F. demonstrou uma boa aptidão para a realização das duas provas. Com relação a atleta E.P. pudemos verificar in loco, que sua performance foi prejudicada mais pela utilização de técnica inadequada do que pela sua condição física. Ao analisarmos os resultados obtidos pela atleta S.G., verificamos que principalmente no arremesso de disco, seu resultado está bem distante do primeiro lugar, mas vale ressaltar que a referida atleta competiu nesta prova em uma classe superior à sua, uma vez que não houve a mesma prova para sua classe. Contudo, é importante salientar, que apesar de estar competindo com classe superior a atleta possui potencial para alcançar melhores resultados desde que busque melhorar sua condição física e aprimorar suas técnicas de acordo com suas potencialidades e limitações.

TABELA 8

Resultados individuais do judô

\begin{tabular}{lccccc} 
Nome & Esporte & Classe & Vitórias & Derrotas & Classif. \\
\hline H.M.A. & Judô & B2 & 0 & 1 & - \\
\hline A.O. & Judô & B1 & 2 & 2 & 5 \\
\hline A.T.S. & Judô & B1 & 3 & 0 & 1 \\
\hline D.D. & Judô & B1 & 0 & 2 & - \\
\hline L.M.F. & Judô & B1 & 1 & 2 & 5 \\
\hline
\end{tabular}

A tabela 8 nos aponta a participação do Brasil na modalidade de Judô para cegos e deficientes visuais, onde a delegação brasileira participou com 05 (cinco) judocas. O atleta A.T.S. Medalha de ouro em Atlanta em 1996, repetiu a sua performance em Sydney 2000 conseguindo novamente a medalha de ouro sem nenhuma derrota. Os demais judocas, apresentaram-se relativamente bem conforme podemos observar os resultados acima, pois dois deles conquistaram o 5o lugar em uma competição de nível extremamente elevado e os outros dois não conseguiram classificação final. Apesar dos bons resultados do Judô brasileiro em eventos internacionais, observamos a necessidade de empenho na renovação de atletas nesta modalidade, pois pudemos observar nos Jogos de Sydney, um aumento considerável do nível dos atletas que se apresentaram na competição.
TABELA 9

Resultados individuais do tênis de mesa

\begin{tabular}{lllccc}
\hline Nome & Esporte & Classe & Vitórias & Derrotas & Classif. \\
\hline L.M. & Tênis mesa & MS11 & 1 & 1 & - \\
\hline C.M. & Tênis mesa & MS6 & 1 & 2 & - \\
\hline F.S. & Tênis mesa & MS1 & 0 & 2 & - \\
\hline L.S. & Tênis mesa & MS3 & 1 & 2 & - \\
\hline A.S. & Tênis mesa & WS11 & 0 & 3 & - \\
\hline
\end{tabular}

TABELA 10

Resultados individuais de ciclismo

\begin{tabular}{lcccccc}
\hline Nome & Prova & Classe & $\begin{array}{c}\text { Tempo } \\
\text { (min.) }\end{array}$ & Classif. & $\begin{array}{c}\text { Vencedor } \\
\text { (min.) }\end{array}$ & $\begin{array}{c}\text { Dif. } \\
(\%)\end{array}$ \\
\hline C.S. & $1 \mathrm{~km}$ & LC2 & & Desq & & - \\
C.S. & $4 \mathrm{~km}$ & LC2 & & Desq & & - \\
C.S. & $63,7 \mathrm{~km}$ & LC2 & 1 LAP & 12 & 105,31 & - \\
\hline R.S. & $1 \mathrm{~km}$ & LC1 & & Desq & & - \\
R.S. & $72,8 \mathrm{~km}$ & LC1 & 119,06 & 15 & 114,08 & 4,2 \\
\hline
\end{tabular}

TABELA 11

Resultados individuais de halterofilismo

\begin{tabular}{lllllll}
\hline \multicolumn{1}{c}{ Nome } & Prova & Classe & $\begin{array}{c}\text { Carga } \\
\text { (kg) }\end{array}$ & Classif. & $\begin{array}{c}\text { Vencedor } \\
\text { (kg) }\end{array}$ & $\begin{array}{c}\text { Dif. } \\
(\%)\end{array}$ \\
\hline João B. & Supino & $75 \mathrm{~kg}$ & 155 & 15 & 235 & 51,6 \\
\hline Alexsander W. & Supino & $82,5 \mathrm{~kg}$ & 157,5 & 14 & 242,5 & 54,0 \\
\hline Terezinha S. & Supino & $56 \mathrm{~kg}$ & 70 & 8 & 120 & 71,4 \\
\hline
\end{tabular}

TABELA 12

Resultados individuais de esgrima

\begin{tabular}{lccccc} 
Nome & Esporte & Classe & Vitórias & Derrotas & Classif. \\
\hline A.M. & Esgrima & B & 0 & 4 & 10 \\
\hline A.M. & Esgrima & B & 0 & 5 & 11 \\
\hline
\end{tabular}

Com relação às modalidades de Tênis de Mesa, Halterofilismo, Esgrima e Ciclismo, como podemos observar nas tabelas acima, o Brasil não se apresentou bem durante os Jogos Paraolímpicos de Sydney. O Tênis de Mesa e o Halterofilismo, são modalidades que o Brasil estreou nos Jo- 
gos Paraolímpicos e talvez a inexperiência possa ter influenciado nos seus resultados. Contudo, são modalidades em crescimento no país e acreditamos que, com um melhor direcionamento nos treinamentos e maior oportunidade de intercâmbio internacional possamos nos apresentar melhor nos Jogos de Atenas. No que diz respeito ao Ciclismo e Esgrima, são modalidades que participaram nos Jogos de Atlanta, mas que ainda necessitam de uma maior divulgação, para massificação da modalidade e como conseqüência buscar uma melhor qualidade.

\section{TABELA 13}

Resultados das modalidades coletivas do Brasil

\begin{tabular}{ccc}
\hline Equipe & Classif. & Total equipes \\
\hline Futebol & 3 & 8 \\
\hline Basquete & 5 & 8 \\
\hline
\end{tabular}

A tabela 13 , nos mostra a classificação das equipes brasileiras nas modalidades coletivas de futebol de paralisados cerebrais e basquete para deficientes mentais. Ao analisarmos simplesmente os números constantes da tabela 5.15 , os mesmos apenas indicam a classificação final do Brasil e o número de equipes participantes dos Jogos. No entanto, é necessário ressaltar que a equipe de futebol do Brasil apresentou uma significativa evolução em relação à sua última participação em Atlanta, onde permaneceu em 6o lugar com oito equipes participantes. Com relação ao basquete, acreditamos que a inexperiência em Jogos Paraolímpicos possa ter influenciado na sua atuação, uma vez que foi sua estréia em uma Paraolimpíada, por outro lado, a grande subjetividade com relação à elegibilidade dos deficientes mentais para participarem dos Jogos, propiciando que atletas sem nenhuma deficiência participassem como deficientes mentais, como foi o caso da equipe da Espanha, pode, também, ter exercido uma certa influência na sua participação.

\section{CONCLUSÃO}

Ao analisarmos os resultados gerais obtidos pelo Brasil nos Jogos Paraolímpicos de Sydney 2000 e compararmos com os resultados anteriores, verificamos que o desporto adaptado no Brasil está em plena evolução, quer seja no seu aspecto administrativo, de marketing e também técnico. As Entidades Nacionais de Desporto para pessoas portadoras de deficiência, têm buscado, principalmente após os Jogos Paraolímpicos de Sydney, uma melhor estruturação e profissionalização administrativa para propiciar um melhor desenvolvimento do desporto a elas vinculados. Por outro lado, os Jogos Paraolímpicos de Sydney foi, também, o grande marco para o Comitê Paraolímpico Brasileiro no que diz respeito à sua solidificação como representante do Desporto Paraolímpico no Brasil.

Os resultados obtidos em Sydney, aliados à ousadia do Comitê Paraolímpico Brasileiro em buscar dar visibilidade ao Desporto Adaptado, propiciaram, através do trabalho de mídia, apresentar à sociedade brasileira um desporto até então desconhecido, possibilitando, desta maneira, um reconhecimento dos feitos dos atletas Paraolímpicos, por parte dos órgãos governamentais, assim como pela população brasileira.

Desta forma, acreditamos que a busca de parcerias com empresas privadas para a sustentação desse desporto em crescimento, seja inevitável e de fundamental importância para a massificação e desenvolvimento desse desporto. Por outro lado, acreditamos, também, que a participação dos centros de excelências das Universidades representam um papel preponderante para propiciar as bases científicas necessárias para o desporto de alto nível, contribuindo com os conhecimentos produzidos, na preparação de profissionais, avaliações físicas, médicas, biomecânicas, recomendações e acompanhamento de treinamento dos atletas brasileiros. Assim, podemos concluir que os horizontes para o desporto paraolímpico do Brasil se vislumbra extremamente otimista para sua preparação e participação em Atenas 2004.

\section{AGRADECIMENTOS}

- Comitê Paraolímpico Brasileiro (CPB)

- Secretaria Nacional de Esportes

- Rede Cenesp

- Associação Fundo de Incentivo à Psicofarmacologia (AFIP)

- Universidade Federal de Uberlândia (UFU)

- Universidade Federal de São Paulo (Unifesp) 